\title{
\#FOODPORN: FETISHIZED SHARING OF FOOD AND ITS IMAGES
}

\author{
Francesco Piluso \\ University of Bologna \\ francesco.piluso2@unibo.it
}

\begin{abstract}
Once translated into images, food acquires a broader meaning. Food is no longer merely something to eat, but to show, share and look at. The increasing amount of images and pictures of dishes on our social networks, associated with hashtags such as \#foodporn, expresses this renewed social, communicative and provocative function of food. However, the exhibition of these images is quite ambivalent when it comes to establishing determined patterns of visual and social relationships with and between users. The aim of this article is to analyze and attempt to provide mediation to this ambivalence. The pornographic exposition of food images no longer presupposes a transitive form of consumption by the user, but becomes pure and self-reflexing spectacle. The images are obscene (Baudrillard [1981] 1994) and characterized by an excess of transparency on their object which abolishes any form of seduction (Baudrillard [1979] 1990). Barthes ([1980]
\end{abstract}


1981) defines this kind of image as unary. Pornographic images are an emblematic example. In terms of their self-evident objectivity, these pictures lack any punctum, any piercing sign of a relationship with or openness to the observer (see Eco 1962; 1979). Nevertheless, behind their apparent transparency, the images are always products of specific perspective cuts, and still able to convey mystery, meaning and involvement. The unary image of food is a further fragment in a series of multiple perspectives on the same object. Such potentiality is actualized in our (social) media culture in which sharing and continuous remediation of images and pictures of food constitute a complex storytelling of the object. This, in turn, fosters further participation by the users. The ambivalence between the indifference of the pornographic image and the involvement in the serialization of the detail is synthetized by the notion of fetishism (Baudrillard [1972] 2019). The social (and) media scenery seems to exemplify and radicalize a sort of commodity fetishism, in which social relationships between users are shaped and mediated by (social) media relationships between images of food.

Keywords: \#foodporn; obscenity; sharing; fetishism

\section{Introduction}

In our contemporary social media arena, the consumption of food goes beyond its nutritional function and material essence to become a broader aesthetical and semiotic object (Stano 2016, esp. 9-26) ${ }^{1}$. Food is no longer merely something to eat, but increasingly a visual element to show, share and look $\mathrm{at}^{2}$. A large volume of images and pictures of dishes on our social networks expresses this renewed social and communicative function of food. However, the exhibition of these images is quite ambivalent when it comes to establishing determined patterns of visual and social relationships with and between users.

Although the traditional indexical and material link with food is progressively being lost, images are still able to create connections with and, especially, between their consumers. The main social indicator of the new understanding of food and of its images is probably the hashtag \#foodporn (Calefato, La Fortuna and Scelzi 2016; Vagni 2017). At the same time, the tool

\footnotetext{
${ }^{1}$ See also Marrone $(2014 ; 2015)$ and Giannitrapani and Ventura Bordenca (2019) for other recent semiotic studies and perspectives on food.

${ }^{2}$ For a deeper insight on the synesthesia of food and the strict relationship between taste and sight see Marrone (2016) and Boutaud (2016).
} 
of the hashatag itself signifies the emergence of a consistent corpus of images (characterized by common features and understandable through same patterns) and the social relevance and value of this corpus. The label "foodporn", instead, allows us to individuate the specific features of the images around which those general categories are formed and social trend is fostered.

In this essay, we will demonstrate that it is precisely the self-evident aspect of the pornographic pictures which questions their role in mediating and fostering the sociality of the users. The total transparency of the image on its object is just an effect of a series of cuts and exclusions. These cuts and exclusions make the single picture an element in a series of multiple point of views and possible narrations on the same object. Therefore, the photographed object continuously reveals and re-veils some of its secret aspects, its expressive potentiality and fascination. Using the notion of fetishism, the aim of this essay is to analyze the semiotic ambivalences of the social production, sharing and consumption of our food images.

\section{From the social scene to media obscenity}

By reporting some of the classic and critical understandings on this object and its relative images, Tito Vagni (2017) retraces the steps that have led to this new social trend related to food (emblematized by the spread of the hashtag \#foodporn). Roland Barthes offers some early semiotic understanding of food as a meaningful element in the socio-cultural structure. Barthes ([1961] 1997) interprets food as "communication system" per se. He does this by highlighting its capacity to talk about its consumers and their social contexts. The author states how food is no longer conceivable only in its specific function as material nourishment, but acquires new values and meanings as a socio-cultural sign.

Jean Baudrillard ([1970] 2017; [1972] 2019) not only follows Barthes' critique on the use-value of food, he also radicalizes its understanding as sign-value. Indeed, Baudrillard challenges Barthes's interpretation of food as a positive sign (value) reflecting a specific social status, in order to highlight the differential and relational logic of this sign. He does this by emphasizing the structural dialogue of food with other commodities and signs (sign-exchange value). Albeit focused on the sign-value of food, Barthes still reads such a value in ideological terms, as reflection and reproduction of the material relationships of power. Baudrillard subverts such a materialist/Marxist perspective by understanding society not as an ideological reflection of material relationships, but as a directly structural system of exchange of differential signs/values. Food is a key element therein. In 
Baudrillard's perspective, food, like any other commodity, acquires its value and meaning only because it is structured as a differential element, sign (or image) in a socio-cultural series. The consumption of these signs and images does not reflect a specific social distinction and ideological values, but the sharing of an entire social code and the consequent formation of social relationships and connections. Nowadays, the exchange of images of food on social media platforms is a way of further reproducing and remediating these patterns of social belonging. The sharing of an experience related to food has to be further shared in the new social media arena, in which the socio-semiotic essence of food is actualized in form of images and relative hashtags:

Every material referent incorporates its own memory and myth, which represent the actual seductive qualities of the objects. The mythicization of reality and its consequent amplification are produced by the modern systems of communication that implement a great work of mediatization of the real through their language. (Vagni 2017: 58, translation mine)

One of the most popular hashtag related to these hyper-mediated images of food is \#foodporn. This label further points out certain ambivalences which question the role of food as reflection and a factor of connection with and between consumers. In order to fully understand such ambiguities, it will be useful to start with Barthes again, and to return to pictures of foods before the era of contemporary social and digital media. In Mythologies (1957), Barthes describes the pictures of food on the magazine Elle. The author points out how every dish seems to be covered by a lucid patina which attracts the gaze of the consumer by increasing the visibility of food. At the same time, it excludes the same consumer by projecting the food into an outer space of imagination and unreality.

Nowadays, food porn images on social media take these features and effects to the extreme. According to Vagni (2017), the hashtag \#foodporn is related to the concept of "obscenity" theorized by Baudrillard $(1990,1994)$. The author describes the advent of the social media arena by emphasizing transition from the scene as space for representation (characterized by a significant distance between the image/object and the spectator/subject) to the obscene (characterized by the excess of proximity to the object) abolishing any gap for interpretation and seduction. The overabundance of food images on our social platforms is metonymically reproduced at the level of each image in terms of overexposure of the related object. This ipervisibility seems to dissolve any surface of separation, any patina, in order to promote 
total transparency. It provides an extreme close-up capable of showing details never perceived by human sight.

The emphasized effect of reality manifests itself as an artificial reproduction which goes beyond reality itself. Porn images project their objects in a sort of hyperreal dimension. The consumption of the image is no longer a transitive and interactive operation, but a mere self-referential spectacle of the image itself. The extreme close-up offered by the porn image burns its own object, its sign-exchange value, by deleting every substantial shadow, every meaningful negativity, and every secret. In other words, pornography makes any form of seduction impossible. Therefore, despite, or better, by means of the same transparency, pornographic images of food radicalize that game of attraction and exclusion seen in Barthes's analysis.

Barthes himself, in his later reflections on photography (1981), offers other interesting insights into pornographic pictures and images. Pornographic pictures are a clear example of what Barthes defines as "unary images",

Nothing more homogeneous than a pornographic photograph. It is always a naive photograph, without intention and without calculation. Like a shop window which shows only one illuminated piece of jewelry, it is completely constituted by the presentation of only one thing: sex: no secondary, untimely object ever manages to half conceal, delay, or distract (Barthes 1981, 41)

Pornographic images lack any punctum (ivi) - i.e. the capacity to impinge upon the observer by opening up a breach of contact with this latter. Their saturation does not permit any openness, any hole to be made by the interpretative cooperation on the part of the reader (see McLuhan 1964; Eco 1979). The evidence of involvement on the part of the observer given by an extreme closeness to the object degenerates in the total indifference, "you have nothing to add, that is to say, nothing to give in exchange" (Baudrillard 1990).

\section{From the detail to the whole}

The advent of the pornographic images of food seems to prevent any possibility of social exchange around these images. However, we live in a historical period where food and its images appear to be key elements in fostering socialization and mediating exchange between users on our media platforms. On the one hand, the pornographic image reduces the degree of complexity of the object by displaying its apparent positivity and integrity. On the other, because of such a reduction, the same picture implies a series of other images and perspectives on the same object, aimed at restoring its 
complexity. As Sontag (1977) states, the total transparency of a picture is always the result of a semiotic cut through the plane of the expression. This inevitably generates exclusions and shadows upon the object.

This dialectic becomes even more evident with regard to digital pictures of food on new media platforms. Users can easily and directly insert their pictures into the social circuit and hence, the image is subjected to comments and further sharings by other users. The single image becomes an element and factor of propulsion for the development of a story about the object. Only the virtual totality of the elements is able to reproduce and remediate the complexity of the object itself. Therefore, the apparent totality and closure which characterizes the unary pornographic image is metonymically related/opened to the wholeness of the object - as realized in the circuit of images driven by the users.

Indeed, the "object" does not coincide with the referent of the image. There is nothing more to say or know about the specific dish of food beyond its pornographic exhibition. The object coincides with the food as a social (media) element that does not exist a priori. It is constantly reproduced and unfolded along the chains of its relative images. Sharing is always a mechanism of abstraction from the materiality and individuality of the referent. Indeed, such media elements no longer have a discrete referent outside the social platform of their exchange. They are directly conceived to be shared in the chain of contents in relation to which they acquire a differential value.

The focus on details, typical of pornographic images, fosters the observers' fetishistic gaze. This can be understood as the particular modality of visualization which cuts through the complex and overall horizon of the object. However, the details recall the wholeness of the object, as a chimeric original condition, which the user can only retrace a posteriori through the collation of its blinding fragments. The user is never fully aware of having an active role in the integration of this series of elements through which the object is actually produced. The transition from serialized detail to the object as a whole seems to find a new reflexive closure. Therefore the object continuously reveals and re-veils its secrets. These images do not stimulate either indifference, or participation, but become objects of a fetishistic gaze and relation. The images attract us by the virtue of their closure and autonomy - the same aspects that, at the same time, exclude us.

In such exclusion, the fetishism that characterizes the consumption of the pornographic image also acquires a Marxist connotation more focused on the alienated production of the image itself. 
Neither the observer, nor the producer of the image is ever fully-aware of his/her active role in the construction of the image. The "subject" (the author of the image, or simply the user who shares the image on his/her personal profile) is alienated and fragmented in the same mechanism of serialization that affects the object.

According to Baudrillard (2017), the rhetorical stress on personality and personalization which characterizes cultural discourse in our consumerist society, mystifies the substitution of a real individual persona with its social simulacrum. This mechanism is further exemplified in our social media platforms which nowadays represent the main field for the social reproduction of individuals. Social platforms today play the role of mediation (and collapse) between the individual sphere of the self and the social arena of self-exposure ${ }^{3}$. Subjectivity is objectified and its absence exorcized by the signs of a generic self without a specific referent - mere differential values within the range of the same model of personalization. Personalized differences and differential personalities are produced in function of a model. Through the sharing of their experiences, objects and pictures, users do not display their own actual individual subjectivity, but are mere bit players in a social game of exchange of signs-differences, with the further risk of a "total anonymity since difference is by definition that which has no name" (ivi: 105).

As in the case of the object, the relational essence which characterizes the category of the subject is exemplified by its collapse into the serialized logic of its digital simulacrum on social media platforms. Even though the simulacrum of subjectivity is inscribed in the shared elements, their value is not in the mise-en-scéne of the individual subject through a sort of link with the element: this represents me, or I produced this. Indeed, what is accomplished by the act of sharing is the need to increase the social (exchange) value of his/her own profile. This can be seen as desire of sociality and belonging in its more mediated and social version, i.e. relationships between subjects mediated by relationships between media images.

\section{From the social exchange to the social sharing (of the code)}

The dissolution of the subject into the autonomous logic of its media images cannot be totally interpreted through the category of alienation into the traditional perspective of commodity fetishism. Indeed, the fetishized value of the object is played around the category of social exchange, along

\footnotetext{
${ }^{3}$ Both in the sense of exposure of the individual self and of exposure of the public/social arena itself.
} 
which both production and consumption converge. In this regard, Baudrillard (2019) offers an interesting insight on the construction of social relationships around the exchange of fetishized objects much before the advent of social media. The author analyzes the process of dépense. During art auctions, participants speculate on the value of works of art, and burn huge amount of money until the explosion in their economic value. In this way, the value of the work of art is no longer a fetishized reflex of the underlying relationships of production (economic exchange-value), but a differential value in relation to the arbitrary code of its own valorization. A manipulation takes place at the moment of the exchange of the object (sign-exchange value). In this performance, it is not important to win the auction, but to be part of the exclusive circle of players who share the code and take part in this manipulation.

The work of art becomes a fetishized object just because, due to its differential value, it reflects and affirms the prestigious role of the participants who share the code for the social valorization of the work itself. The wasteful expenditure of money is not aimed at satisfying the exigency to express one's own singular individuality through the exclusive possession of the work of art, but it satisfies the need for social conformity, sharing, exchange amongst peers. ''Object Fetishism' never supports exchange in its principle, but the social principle of exchange supports the fetishized value of the object" (ivi: 112)

Through the analysis of the process of dépense, Baudrillard goes beyond the traditional Marxist critique of the political economy. He reinterprets its categories and mechanisms as particular moments within a generalized principle of social exchange of differences. The author demonstrates how relations between subjects are not mediated by relations between material products (and their economic value that masks relations of production), but by abstract objects, signs (and their differential value that masks relations of signification and exchange). In this fetishism, the sign-value is not a positive attribute of the commodity. It is not an ideological substitute of its material use-value, aimed at sustaining exchange and consequently the entire cycle of production. Rather, the sign-value is a differential element intrinsic in the commodity form as exchange-value.

Nowadays, the social sharing of food and its images on social media represents a modern, further rationalized, version of the principle exchange in the process of dépense. Nevertheless, certain key differences emerge from the homology between these two processes. Dépense is a mechanism aimed at exorcizing competition and the emergence of an individual subject by establishing a circle of aristocratic peers. However, the same wasteful ex- 
penditure is still a very performative and situated performance. It is a challenge to the abstract logic of economic exchange. On the other hand, in contemporary forms of image sharing, subjectivities are automatically reproduced as codified differences within an abstract model which radicalize the fetishized relationship with their own images.

Consumption (and exchange) of food (and related images) is a very emblematic example of pointing out these contemporary forms of dépense. Experiences of consumption at a fancy restaurant signify participation in a prestigious social scene. The sharing of such a privilege needs to be further shared on social media networks, in order to demarcate our "aristocratic" practice and role. Nevertheless, it is the same act of demarcation and remediation of social prestige that reduces the subject to a mere objectified difference in the series of images, pictures, and profiles which characterize our social arena.

Even when the object of the image is not the experience of consumption of a meal in a trendy restaurant, but the personal production of a tasty dish, its sharing is not exempt from the same fetishistic pattern. Once translated into the form of image-sign, food loses its contingent, indexical relationship with its producer. The presence of a picture on a personal profile page is only a necessary "encoded index" (Baudrillard 2019: 99) according to the architecture of the platform, where the specific content is marked and interpreted as "personal". The automatic reproduction of the operative logics of the platform is hidden behind this simulation of the self. There could be no shared personal content without an underlying shared code. In this regard, it is no coincidence that our images are often defined by hashtags. They are not only hypercodified signs of sharing of a specific social tendency, but also as operational dispositifs of sharing and reproduction of the social media code (see Piluso 2019). Hashtags exemplify and actualize the fetishistic translation of our sociality in a series of operational elements within the increasingly self-reflexive social media arena.

\section{Conclusions}

We have outlined here some of the processes which characterize our sociality in contemporary media culture by analyzing of some of its most emblematic elements: images of food. These images, as objects of sharing, have a key role in shaping social relationships between users on new media platforms. The transition from food to its digital images indicates the signifying and communicative function which food has always had beyond its material use-value, by taking its fetishistic effect to extremes. The pornographic style of many of these pictures fosters a fetishistic gaze on details 
that, despite their total transparency on the object, imply chains of other elements and perspectives on the same object. These chains of elements, in turn, reflect and reinforce a fetishized serial mechanism which characterizes the general exchange of our contents and profiles on new social media platforms. Despite or due to their involvement in these chains of sharing, the same users are reduced to relational and operative elements within the structure of social media. Indeed, the sharing of an image implies the sharing of the code in which that image acquires its differential value. Its value becomes fetishized, since it is able to reflect the relational (exchange) value of the subject in that social structure.

That is why the dispositif of the hashtag is so emblematic. Its use expresses the differential value of the single image as part of a social trend and signifies the involvement of the user in the same social trend. At the same time, besides the single specificity of each social trend, the hashtag itself crystallizes the user's sharing of the code of the social platform. In its self-reflexivity, the hashtag becomes an operative tool for the increasing fetishization of our social relationships. Therefore, \#foodporn represents a perfect synthesis with its apparent internal dialectic between the particular features (foodporn), and the general trend (\#), as well as the detail (the pornographic/unary image) and the whole (the active sharing). Social exchange focused upon food has never been so fetishized as in the social sharing of its pornographic images.

\section{Bibliography}

Barthes, R. 1957. Mythologies. Paris Editions du Seuil.

Barthes, R. 1997 [1961]. Toward a Psychosociology of Contemporary Food Consumption. In Counihan, C., P. van Esterik (eds.). Food and Culture: A Reader. New York \& London: Routledge, 20-27.

Barthes, R. 1981. Camera Lucida. New York: Hill and Wang.

Baudrillard, J. 1990. Seduction. Montreal: New World Perspective.

Baudrillard, J. 1994. Simulacra and Simulation. Ann Arbor: The University of Michigan Press.

Baudrillard, J. 2017. The Consumer Society. Myths and Structure. London: Sage. 
Baudrillard, J. 2019. For a Critique of the Political Economy of the Sign. London: Verso.

Boutaud, J.-J. 2016. "L'esthésique et l'épiphanique: Traces figuratives de la saveur”. Semiotica, No. 211, 203-229.

Calefato, P., L. La Fortuna and R. Scelzi. 2016. "Food-ography: Food and New Media”. Semiotica, Issue 211, 371-388.

Eco, U. 1962. Opera aperta. Milano: Bompiani.

Eco, U. 1979. Lector in fabula. Milano: Bompiani.

Giannitrapani, A., B. I. Ventura (eds.). 2019. Politiche della cucina: Discorsi, conflitti, culture. E/C. Rivista dell'Associazione Italiana di Studi Semiotici, Anno XIII, n. 27. Available at: https://iris.unipa.it/retrieve/handle/10447/468021/1068213/2020_mangano_EC_il_senso_del_dolce.pdf.

Marrone, G. (ed.). 2014. Gatromania. Milano: Bompiani.

Marrone, G. (ed.). 2015. Buono da pensare. Cultura e comunicazione del gusto. Roma: Carocci.

Marrone, G. 2016. "Food Meaning: From Tasty to Flavorful". Semiotica, No. $211,187-201$.

McLuhan, M. 1964. Understanding media. New York: McGraw-Hill.

Piluso, F. 2019. "Lo Stereotipo come Modo di (ri)Produzione e Distruzione Segnica: Esempi e Strategie di Sovversione”. Filosofi(e)semiotiche, Vol. 1, No. 6, 183-195.

Sontag, S. 1977. On Photography. New York: Farrar, Straus and Giroux.

Stano, S. (ed.). 2016. Semiotics of food. [Special issue]. Semiotica, No. 211.

Vagni, T. 2017. "Food is (not) porn, le immagini del cibo e l'orizzonte della scomparsa”. Mediascapes, No. 9, 56-69. 